\title{
Essais
}

Revue interdisciplinaire d'Humanités

$12 \mid 2017$

Textes et contextes : entre autonomie et dépendance

\section{Du texte et de la diversité de ses entours}

Jean-Paul Bronckart

\section{(2) OpenEdition}

Journals

Édition électronique

URL : http://journals.openedition.org/essais/3158

DOI : 10.4000/essais.3158

ISSN : 2276-0970

Éditeur

École doctorale Montaigne Humanités

Édition imprimée

Date de publication : 15 juin 2017

Pagination : 211-229

ISBN : 979-10-97024-02-4

ISSN : $2417-4211$

Référence électronique

Jean-Paul Bronckart, « Du texte et de la diversité de ses entours », Essais [En ligne], 12 | 2017, mis en ligne le 12 octobre 2020, consulté le 25 octobre 2020. URL : http://journals.openedition.org/essais/ 3158 ; DOI : https://doi.org/10.4000/essais.3158 


\section{Du texte et de la diversité de ses entours}

\section{Jean-Paul Bronckart}

Conformément aux objectifs assignés aux contributeurs de ce numéro, nous proposerons dans ce qui suit un examen du statut et des rapports qu'entretiennent le texte et le contexte, en saisissant la seconde notion dans son acception la plus large qui, comme l'indique notre titre, est celle d'un « entour " constitué d'une diversité d'entités potentiellement influentes.

L'émergence de la notion de " contexte " parait logiquement indissociable de celle de " texte ", et dans un premier temps nous montrerons que si l'acception de sens commun de ce second terme paraît évidente, les sciences du langage ont éprouvé - et éprouvent encore - quelque peine à lui accorder un signifié précis. Nous examinerons ensuite quelques approches théoriques qui, au cours du XX $\mathrm{XX}^{\mathrm{e}}$ siècle, ont visé à conceptualiser les ingrédients du contexte et les modalités de leurs interactions avec les textes. Nous présenterons alors notre propre conception des rapports entre les textes et leurs entours, en prenant appui sur quelques cadres théoriques dont nous nous inspirons en permanence (en l'occurrence ceux de Coseriu, Saussure, Volochinov et Vygotski), puis en présentant sur ces bases un appareil conceptuel visant, aussi modestement que l'on voudra, à la cohérence et à la complétude.

\section{L'objet « texte », mal-aimé des sciences du langage}

Dans le langage courant, le terme de " texte » désigne une unité de production verbale écrite identifiable aux marques visuelles de son bornage, et à ce même plan du langage usuel, ce terme tend aujourd'hui à désigner également les unités de production verbale orale, dont les bornes, auditives et temporelles, sont toutefois moins aisément identifiables. En dépit du fait que ce sont les exemplaires de ces unités de production verbale qui constituent le matériau premier auquel s'adressent les sciences du langage, la notion de " texte " n'a été que tardivement mobilisée dans lesdites sciences et y demeure relativement peu usitée en raison de l'indécision subsistant quant au statut théorique de l'objet auquel elle s'adresse. 
En amont historique des sciences du langage, s'était déployé comme on le sait un vaste mouvement d'étude des œuvres poétiques et/ou littéraires, dont la genèse symbolique se situe dans ceux des enseignements de Platon (La République) et d'Aristote (Poétique et La Rhétorique) qui proposaient des classements des sortes d'œuvres. Dans la plupart des traductions contemporaines de ces ouvrages, celles-ci ont été qualifiées de " genres " poétiques ou littéraires, mais Genette a magistralement démontré que les classements de l'Antiquité (comme plus tard ceux des XVII ${ }^{e}$ et XVIII ${ }^{e}$ siècles) portaient en réalité sur des différences de modes d'énonciation, plutôt que sur des différences proprement génériques :

[...] ce qu'Aristote appelle "façon d'imiter " équivaut strictement à ce que Platon nommait lexis: nous n'en sommes pas encore à un système de genres; le terme le plus juste pour désigner cette catégorie est sans doute bien celui, employé par la traduction de Hardy, de mode : il ne s'agit pas à proprement parler de "forme" au sens traditionnel, comme dans l'opposition entre vers et prose, ou entre les différents types de vers, il s'agit de situations d'énonciation. ${ }^{1}$

Viëtor a confirmé ce diagnostic du statut fondamentalement énonciatif des classements établis dans la tradition littéraire, en s'appuyant sur les propositions formulées par Goethe dans le Divan oriento-occidental :

Goethe n'[...] emploie absolument pas la notion de " genre » (Gattung) mais il nomme la ballade, l'épigramme, le récit, l'ode, la satire, etc., des " espèces poétiques "(Dichtarten), et, pour cette raison, l'épopée, la poésie lyrique et le drame des "formes naturelles (Naturformen) de la poésie »:

$<$ Il n'y a que trois véritables formes naturelles de poésie: l'une qui raconte clairement, une autre qui s'exalte et s'enthousiasme, une troisième qui agit personnellement. Ces trois modes poétiques peuvent agir ensemble ou séparément $[\ldots]>3$.

Dans l'ensemble de ces approches, sauf cas d'usage prosaïque, la notion de " texte" n'est pas mobilisée, ce qui semble tenir notamment au fait que les "modes" ou "formes naturelles " qui y sont visés ont un empan qui en principe ne correspond pas à celui du texte comme production verbale bornée ; plusieurs modes énonciatifs différents peuvent en effet se succéder sans dommage dans une même production verbale. Ce type d'absence se prolongera dans de nombreux cadres théoriques du $\mathrm{XX}^{\mathrm{e}}$ siècle et sera particulièrement remarquable dans les œuvres de Benveniste et de Foucault. S'agissant du premier auteur, le terme de « texte » est totalement absent de l'article sur Les relations de temps qui illustre pourtant l'importante distinction entre productions verbales relevant de l'ordre du discours $v s$ de l'ordre de l'Histoire ; l'ar-

1 Gérard Genette, "Introduction à l'architexte ", in Gérard Genette et al., Théorie des genres, Paris, Éditions du Seuil, 1986, p. 98.

2 Johann Wolfgang von Goethe, Le divan oriento-occidental, Paris, J.-A. Merklein, 1835.

3 Karl Viëtor, "L'histoire des genres littéraires ", dans G. Genette et al., Théorie des genres, Paris, Éditions du Seuil, 1986, p. 10-11. 
ticle sur Les niveaux de l'analyse linguistique évoque comme niveau ultime de structuration langagière "le discours actualisé en phrases " et ne mentionne nullement le terme de "texte», et celui-ci, en conséquence, n'apparait pas dans l'index des deux volumes des Problèmes de linguistique générale ${ }^{4}$. Dans l'Archéologie de Foucault, le terme de texte n'est jamais mobilisé comme notion théorique, mais dans la tentative réalisée par l'auteur pour " fixer le vocabulaire " en ce domaine, il semble y substituer le terme d' "énoncé ", avec cependant une réserve révélatrice du peu d'intérêt qu'il y aurait, selon lui, à saisir les productions verbales dans leur empirique nudité :

[...] on appellera phrase ou proposition les unités que la grammaire ou la logique peuvent reconnaître dans un ensemble de signes [...] On appellera énoncé la modalité d'existence propre à cet ensemble de signes : modalité qui lui permet d'être autre chose qu'une série de traces, autre chose qu'une succession de marques sur une substance, autre chose qu'un objet quelconque fabriqué par un être humain ; modalité qui lui permet d'être en rapport avec un domaine d'objets, de prescrire une position définie à tout sujet possible, d'être situé parmi d'autres performances verbales, d'être doté enfin d'une matérialité répétable. ${ }^{5}$

C'est dans l'œuvre de Coseriu, et plus particulièrement dans son article Détermination et entours ${ }^{6}$, que l'on trouve ce qui semble constituer la première conceptualisation scientifique moderne donnant un statut précis au "texte ". L'appareil notionnel de cet auteur pose comme objet général de la linguistique, l'activité de parler, activité humaine universelle qui se réalise avec les ressources spécifiques d'une langue naturelle ; il pose ensuite le discours, comme acte (ou série d'actes) accompli par un individu dans telle ou telle circonstance donnée ; il pose enfin que le produit de l'activité de parler humaine réside dans la " totalité des textes " et que les " textes" constituent les produits empiriques des actes individuels de discours. Un élément important de cette approche est de considérer que ces produits historiques que sont les textes, loin de constituer les états terminaux, secondaires et volatiles, des processus langagiers, sont au contraire destinés à s'inscrire dans le réseau des ressources de la langue et d'y fournir des modèles exploitables lors de prochains actes de discours.

Dans ses écrits fondateurs du courant de linguistique fonctionnelle, Halliday déclarera pour sa part que l'unité de base de cette discipline "n'est pas le mot ni seulement la phrase, mais le texte ${ }^{7}$, prise de position qu'il renouvellera constamment et fermement dans ses études sur les mécanismes

4 Émile Benveniste, Problèmes de linguistique générale, t. 1 et 2, Paris, Gallimard, 1966 et 1974.

5 Michel Foucault, Láarchéologie du savoir, Paris, Gallimard, 1969, p. 140.

6 Eugenio Coseriu, "Détermination et entours ", dans L'homme et son langage, Louvain, Peeters, 2001, p. 31-67. C'est à cet auteur que nous empruntons le terme d' " entour " mobilisé dans notre titre, terme dont il affirmait (p. 39) qu'il l'utilisait dans le sens donné par K. Bühler au terme allemand Umfeld.

7 Michael Alexander Kirkwood Halliday, "Language structure and language function ", in New Horizons in linguistics, éd. John Lyons, Harmondsworth, Penguin, 1970, p. 160. 
de cohésion organisant la textualité : "For a linguist, to describe language without accounting for text is sterile; to describe text without relating to language is vacuous ${ }^{8}$ ». C'est en référence à cette prise de position de Halliday que Slakta a proposé d'introduire la notion de "texte " dans le champ de la linguistique francophone. Dans un premier article, il soutenait que cette notion devait se substituer à celle de " corpus " utilisée dans l'analyse de discours inspirée de Harris, en précisant toutefois que ledit texte devait être défini " comme action verbale et comme matrice dans lequel la langue prend sens " et que "l'objet-texte " sur lequel avait à travailler le linguiste constituait " un concret-de-pensée, et non [un] concret-réel justiciable de la seule description " ". Cet auteur prônait ainsi l'élaboration d'une linguistique qui certes devait s'adresser aux textes, mais en tant que ceux-ci « entretiennent des rapports avec l'extralinguistique "; position non dénuée d'ambiguïté comme le confirmera la formule bien connue issue d'un de ses textes ultérieurs selon laquelle « le discours, c'est le texte avec ses conditions de production et d'interprétation, et le texte, c'est le discours abstrait de ces mêmes conditions ${ }^{10}$ ". L'approche de Slakta a donné naissance au courant de linguistique textuelle francophone dont la figure de proue est Jean-Michel Adam qui, après avoir longtemps adhéré à la formule ci-dessus rappelée, a récemment clarifié sa démarche en affirmant qu'elle relevait d'une " analyse textuelle des discours " et a re-défini comme suit ses concepts de base :

Tout texte est la trace langagière d'une interaction sociale, la matérialisation sémiotique d'une action sociohistorique de parole [...]. Les genres, organisés en systèmes de genres, sont des patrons sociocommunicatifs et sociohistoriques que les groupes sociaux se donnent pour organiser les formes de langue en discours. Dès qu'il y a texte, c'est-à-dire reconnaissance du fait qu'une suite verbale ou verbo-iconique forme une unité de communication, il y a effet de généricité, c'est-à-dire inscription de cette suite d'énoncés dans une classe de discours. ${ }^{11}$

Dans un dernier courant enfin, le texte est conçu comme un objet autonome, ou tendant à l'autonomie, c'est-à-dire est saisi dans ses propriétés linguistiques observables, indépendamment de ses rapports présumés à l'extralinguistique ou au nébuleux univers du " discours». Dans le cadre de ce type d'approche, c'est à cette entité " texte empirique » qu'est attribuée la dimension de généricité, comme c'est le cas dans la plupart des travaux de la textlinguistik allemande ${ }^{12}$, et ce pour des raisons que Schaeffer avait clairement identifiées :

8 Michael Alexander Kirkwood Halliday, "Systemic background ", in Systemic Perspectives on Discourse, vol. 1, James D. Benson \& William S. Greaves (eds), Norwood, NJ, Ablex Publishing Corporation, 1985, p. 10.

9 Denis Slakta, «Esquisse d'une théorie lexico-sémantique : Pour une analyse d'un texte politique (Cahiers de doléances) ", Langages, n 23, 1971, p. 111.

10 Denis Slakta, "L'ordre du texte ", Études de linguistique appliquée, n 19, p. 30-42.

11 Jean-Michel Adam, La linguistique textuelle, Paris, Armand Colin, 2011, p. 33.

12 Voir Elisabeth Gülich, \& Wolfgang Raible, Textsorten, Differenzierungskriterien aus linguisti- 
[...] d'une manière ou d'une autre le terme de " genre " semble bien être le corrélat à définir de cet autre terme, supposé connu celui-là, que serait le " texte ${ }^{13}$

Un avantage certain d'une définition purement textuelle de la généricité réside dans le fait qu'elle permet d'établir un critère empirique, ce qui n'est pas le cas des théories ontologiques où les genres sont par définition transcendants à la textualité et donc du même coup empiriquement inattaquables. ${ }^{14}$

\section{De quelques approches du contexte}

\section{Les paramètres des situations de communication}

Le texte de Lev Jakubinski intitulé Sur la parole dialogale a ouvert à une approche saisissant les faits langagiers dans leur dimension fondamentalement interactive, dans le cadre toutefois d'une orientation de type behavioriste. Pour cet auteur, le langage est " une variété du comportement humain » et donc une entité à la fois psychologique (dépendant des capacités de l'organisme) et sociologique (dépendant des interactions en cours dans la vie collective). Et c'est sous ce dernier angle qu'il a développé une conception déterministe des effets qu' exercent certains facteurs externes sur les comportements verbaux :

L'activité langagière de l'homme est un phénomène multiforme [...] qui est déterminé par la diversité complexe des facteurs qui ont la parole humaine pour fonction. Sans prendre en compte tous ces facteurs et sans étudier les manifestations multiformes de la parole qui leur correspondent fonctionnellement, il est impossible d'étudier une langue comme phénomène donné directement à la perception vivante, ni d'en mettre à jour la genèse et l'histoire ${ }^{15}$.

Selon Jakubinski, ces facteurs seraient de trois ordres :

- les conditions de la communication dans un ou des milieux habituel(s) [ou] inhabituel(s);

- les formes de communication (médiatisée ou non, unilatérale ou en alternance);

- les buts de la communication verbale et du processus d'énonciation.

Mais l'auteur indiquait toutefois qu'on ne disposait à son époque d'aucune étude permettant de mesurer l'impact effectif, sur les propriétés des énoncés, des trois types de facteurs qu'il avait identifiés.

scher Sicht, Frankfort, Athenäum, 1972 ; Horst Isenberg, " "Text” versus "Satz” » in Probleme der Textgrammatik II, František Daneš \& Dieter Viehweger (eds), Berlin, Akademie Verlag, 1977, p. 119-145 ; Egon Werlich, Typologie der Texte, Heidelberg, Quelle \& Meyer, 1975.

13 Jean-Marie Schaeffer, "Du texte au genre. Notes sur la problématique générique », in Théorie des genres, Gérard Genette (éd.) et al., Paris, Éditions du Seuil, 1986, p. 180.

14 Jean-Marie Schaeffer, op. cit., p. 186-187.

15 Lev Jakubinski, "Sur la parole dialogale ", in Lev Jakubinskij, une linguistique de la parole (URSS, années 1920-1930), Irina Ivanova (éd.), Limoges, Lambert-Lucas, 2012, p. 57. 
Même si elle s'adosse à un positionnement épistémologique différent, d'ordre subjectiviste, l'analyse développée par Benveniste dans L'appareil formel de l'énonciation ${ }^{16}$ présente également un caractère paramétrique, en ce qu'elle identifie un sous-ensemble de marques spécifiques des énoncés renvoyant à des propriétés de l'acte même d'énonciation; comme on le sait, il s'agit des marques déictiques (pronoms personnels, adverbes de temps ou de lieu, ostensifs) dont la valeur ne peut être fixée que par prise en considération de ces paramètres de l'acte langagier que sont le couple émetteur/récepteur, les lieux et temps de l'énonciation, ainsi que tout référent potentiel inscrit dans ce même espace-temps.

On pourrait considérer encore que la théorie des Actes de langage, telle que Searle l'a développée, comporte une dimension paramétrique qui n'est pas thématisée comme telle mais qui sous-tend néanmoins l'analyse que propose l'auteur des effets qu'exercent les faits institutionnels sur la signification " véritable $»$ des actes langagiers :

Tout fait institutionnel repose sur une règle (un système de règles) de la forme " $\mathrm{X}$ revient à $\mathrm{Y}$ dans la situation $S$ ». Notre hypothèse selon laquelle parler une langue c'est accomplir des actes conformément à des règles constitutives, nous amène à poser l'hypothèse suivante : le fait d'accomplir un certain acte, comme par exemple, faire une promesses, est un fait institutionnel. ${ }^{17}$

Alors que l'analyse de Benveniste proposait la lecture d'un contexte constitué de paramètres proprement physiques (l'émetteur, le récepteur et leur ancrage dans l'espace-temps), l'analyse de Searle propose, au travers des multiples exemples qui y sont traités, la lecture d'un contexte constitué de paramètres sociaux, dont celui du cadre institutionnel au sein duquel se déroule un acte langagier, et celui des rôles que peuvent assumer les interactants dans ledit cadre.

\section{L'amont discursif et praxéologique}

Dans L'ordre du discours, Foucault avait indiqué que le travail qu'il avait entrepris, en particulier dans L'archéologie du savoir, relevait essentiellement de l'analyse des effets qu'exercent, sur les productions verbales, non les faits institutionnels en eux-mêmes, mais des systèmes de normes semi-implicites élaborées dans le cours de l'activité verbale :

Voici l'hypothèse que je voudrais avancer, ce soir, pour fixer le lieu [...] du travail que je fais : je suppose que dans toute société la production du discours est à la fois contrôlée, sélectionnée, organisée et redistribuée par un certain nombre de procédures qui ont pour rôle d'en conjurer les pouvoirs et les dangers, d'en maîtriser l'événement aléatoire, d'en esquiver la lourde, la redoutable matérialité. ${ }^{18}$

16 Émile Benveniste, «L'appareil formel de l'énonciation ", Langages, $n^{\circ} 17,1970$, p. 12-18.

17 John Searle, Les actes de langage. Essai de philosophie du langage, Paris, Hermann, 1972, p. 93.

18 Michel Foucault, L'ordre du discours, Paris, Gallimard, 1971, p. 10-11. 
Ce qui est analysé ici, ce ne sont certes point les états terminaux du discours ; mais ce sont les systèmes qui rendent possibles les formes systématiques dernières; ce sont des régularités préterminales par rapport auxquelles l'état ultime, loin de constituer le lieu de naissance du système, se définit plutôt par ses variantes. ${ }^{19}$

La source de ce type d'effet exercé sur les productions verbales concrètes a été qualifiée le plus souvent de "formation discursive ", mais parfois aussi de "pratique discursive" :

Ce qu'on appelle "pratique discursive " peut maintenant être précisé... c'est un ensemble de règles anonymes, historiques, toujours déterminées dans le temps et l'espace qui ont défini à une époque donnée, et pour une aire sociale, géographique ou linguistique données, les conditions d'exercice de la fonction énonciative. ${ }^{20}$

Et l'École française d'analyse du discours a globalement repris et reformulé ce type d'approche, comme en attestent notamment les multiples prises de position de Maingueneau :

Il s'agira ici d'opposer un système de contraintes de bonne formation sémantique (la formation discursive) à l'ensemble des énoncés produits conformément à ce système (la surface discursive). [...] Nous userons du terme " discours" pour référer à la relation même qui unit les deux concepts précédents. ${ }^{21}$

Dans le cadre d'une démarche voisine, qualifiée de « sémiotique des textes ", François Rastier a élaboré un appareil notionnel posant comme instance langagière majeure le " discours ", défini comme pratique verbale articulée aux pratiques sociales, ou encore comme un " usage de la langue normé par une classe de pratiques sociales participant d'une même sphère d'activitée "2 ". Dans cette approche, la notion de "genre » désigne " un programme de prescriptions positives ou négatives, et de licences ${ }^{23}$ ", qui fait écho à la diversification des pratiques sociales au sein d'une même sphère d'activité :

Chaque pratique sociale se divise en activités spécifiques auxquelles correspond un système de genres en coévolution. [...] les textes sont configurés par les situations concrètes auxquels ils participent ; en outre par la médiation des genres et les discours, ils s'articulent aux pratiques sociales dont les situations d'énonciation et d'interprétation sont des occurrences. ${ }^{24}$

Pour Rastier, ce sont donc les genres de discours, en tant qu'ils sont articulés à des genres d'activités humaines, qui exercent une influence " contraignante » sur l'allure d'ensemble des productions verbales.

19 Michel Foucault, L'archéologie du savoir, Paris, Gallimard, 1969, p. 101.

20 Michel Foucault, L'archéologie du savoir, op. cit., p. 153-154.

21 Dominique Maingueneau, Genèse du discours, Bruxelles, Mardaga, 1984, p. 10.

22 François Rastier, "Le langage comme milieu : des pratiques aux œuvres ", Texto !, 2003. Disponible sur : <http://www.revue-texto.net/Inedits/Rastier/Rastier_Langage.pdf>.

23 François Rastier, Sens et textualité, Paris, Hachette, 1989, p. 37.

24 François Rastier, Arts et sciences du texte, Paris, PUF, 2001, p. 228. 
Les deux approches qui viennent d'être convoquées ont en outre en commun une conception bijective des niveaux d'organisation des productions langagières d'un côté, et des facteurs socio-praxéologiques d'un autre côté. Maingueneau affirme par exemple que l'analyse de discours n'a pour objet ni l'organisation textuelle ni les divers composants des situations de communication, mais "le dispositif d'énonciation qui lie une organisation textuelle et un lieu social déterminé 25 ", ces deux niveaux étant fondamentalement associés ou encore ne constituant que les deux faces d'une même médaille, comme il le déclarait dans un ouvrage antérieur :

On admettra [...] qu'il n'existe pas de rapport d'extériorité entre le fonctionnement du groupe et celui de son discours, qu'il faut penser d'emblée leur intrication. Autrement dit, il est nécessaire d'articuler les contraintes qui rendent possible la formation discursive et celles qui rendent possible le groupe puisque ces deux instances sont emportées par la même logique. On ne dira donc pas que le groupe gère de l'extérieur un discours mais que linstitution discursive possède en quelque sorte deux faces, l'une sociale, l'autre langagière. ${ }^{26}$

Dans une perspective analogue, Rastier a soutenu que le domaine praxéologique et le domaine linguistique avaient le même mode de structuration, et qu'à chacun des niveaux de cette structuration les entités des deux domaines sont dans un rapport de correspondance bi-univoque.

Au sein des champs pratiques, les pratiques spécifiques correspondent à des genres ; aux cours d'action, qui sont les occurrences de ces pratiques, correspondent des textes oraux ou écrits. Soit :

\begin{tabular}{|c|c|c|c|c|}
\hline${ }^{27}$ Praxéologie & $\begin{array}{l}\text { Domaine } \\
\text { d'activité }\end{array}$ & $\begin{array}{l}\text { Champ } \\
\text { pratique }\end{array}$ & Pratique & $\begin{array}{c}\text { Cours } \\
\text { d'action }\end{array}$ \\
\hline Linguistique & Discours & $\begin{array}{l}\text { Champ } \\
\text { générique }\end{array}$ & Genre & Texte \\
\hline
\end{tabular}

Ces auteurs procèdent donc par projection, sur les champs de l'extralangagier, de notions élaborées dans le champ linguistique (c'est le cas de la notion de "genre " en particulier), et postulent en outre l'existence d'une correspondance biunivoque entre entités contextuelles et entités langagières. Ces deux thèses nous paraissent infondées, notamment parce que les genres de textes changent nécessairement avec le temps, et qu'à l'instar des autres œuvres humaines, ils sont susceptibles de se détacher des motivations qui les ont engendrés pour s'autonomiser et devenir ainsi disponibles pour l'expression d'autres finalités.

25 Dominique Maingueneau, L'analyse du discours, Paris, Hachette, 1997, p. 13.

26 Dominique Maingueneau, Nouvelles tendances en analyse du discours, Paris, Hachette, 1987, p. 39.

27 François Rastier, Arts et sciences du texte, Paris, PUF, 2001, p. 231. 


\section{Une prise d'appuis théoriques}

\section{L'approche historico-culturelle de Vygostki}

Les travaux que nous réalisons pour notre part s'inscrivent dans la perspective historico-culturelle ouverte par le psychologue Vygotski il y a bientôt un siècle ${ }^{28}$ et dont nous ne pourrons présenter ici que quelques traits majeurs. Le plus important d'entre eux, justifiant l'appellation attribuée à ce courant, est le principe selon lequel l'analyse des conditions d'émergence et de développement des capacités proprement humaines doit s'effectuer en prenant d'abord en considération les effets de plus de 150000 ans d'histoire sociale. Et ce sans sous-estimer pour autant le rôle de l'équipement bio-cognitif hérité, mais en visant au contraire à mettre en évidence les processus par lesquels les capacités et connaissances construites au cours de l'histoire ont pris en charge et ont réorganisé cet équipement inné. La thèse plus spécifique de Vygotski est que c'est l'appropriation et l'intériorisation du langage qui provoquent cette transformation décisive ; ce serait sous l'effet de ces deux processus que le psychisme du jeune enfant, qui fonctionnait jusque là selon des modalités analogues à celles des autres espèces animales supérieure, se trouve entièrement réorganisé par les signes et les structures langagières, et en conséquence s'inscrit désormais dans un mode de fonctionnement d'ordre sociohistorique:

La pensée de l'enfant [...] dépend dans son développement de la maîtrise des moyens sociaux de la pensée, c'est-à-dire dépend du langage. [Avec l'émergence de la pensée verbale], le type même de développement s'est modifié, passant du biologique au sociohistorique ${ }^{29}$.

Pour tenter de valider cette thèse, l'auteur a réalisé un ensemble de recherches sur les processus d'acquisition du langage par l'enfant, qui lui ont permis de montrer d'abord que les significations des mots sont à la fois fondamentalement sociales et fondamentalement dynamiques (elles se transforment en permanence au cours de l'ontogenèse), ensuite que ces significations ne sont pas de l'ordre de l'objet, mais du processus; enfin que les signes intériorisés sont littéralement constitutifs de la pensée consciente :

Le mot est bien dans la conscience ce qui, selon Feuerbach, est absolument impossible à l'homme seul, mais possible à deux. C'est l'expression la plus directe de la nature historique de la conscience humaine. [...] Le mot doué de sens est un microcosme de la conscience humaine ${ }^{30}$.

28 Lev Semionovitch Vygotski, La signification historique de la crise en psychologie, Paris, Delachaux et Niestlé, 1999 ; réédition, Paris, La Dispute, 2010 ; rédigé en 1927.

29 Lev Semionovitch Vygotski, Pensée et langage, Paris, La Dispute, 1997, p. 187 ; édition originale en langue russe, 1934.

30 Lev Semionovitch Vygotski, Pensée et langage, op. cit., p. 500. 
En dépit de la justesse de leur orientation, les propositions qui viennent d'être résumées posent quelques problèmes, découlant en particulier du caractère relativement limité des connaissances qu'avait Vygotski des théoriques et techniques de la linguistique. Et pour poursuivre la démarche de cet auteur, il convient dès lors de la préciser et compléter en sollicitant les apports de linguistes ayant adopté une position épistémologique compatible.

\section{Les apports de Saussure et de Coseriu}

Comme nous l'avons montré ailleurs ${ }^{31}$, depuis la publication de divers manuscrits saussuriens, l'œuvre réelle de cet auteur est en voie de reconstitution et fait apparaître un ensemble d'analyses fondatrices, dont deux sont essentielles pour le thème de la présente contribution.

La première concerne le statut même des signes, tel qu'il est présenté dans La double essence du langage $e^{32}$ et que l'on peut résumer drastiquement comme suit :

- les signes sont des entités bi-face dont les deux versants sont psychiques, c'est-à-dire constitués non d'entités matérielles mais d'images mentales ;

- ces images n'émergent que de leur " accouplement ", dans le processus même de leur union en un signe ;

- les propriétés de la face signifiante du signe ne dépendent nullement de celles de la face signifiée, mais sont établies par pure convention sociale au sein de chaque idiome.

Le développement de cette analyse dans le manuscrit ci-dessus cité (une centaine de feuillets de notes) démontre en fait que les signes verbaux (ne) constituent (que) des cristallisations psychiques d'unités d'échange social, et confirme en conséquence (indirectement) la thèse vygotskienne évoquée plus haut du rôle décisif de l'appropriation des signes dans la constitution d'un psychisme conscient. Cette analyse doit en outre être complétée par une prise de position au moins aussi importante qu'illustre une brève formule maintes fois citée : "Sémiologie = morphologie, grammaire, syntaxe, synonymie, rhétorique, stylistique, lexicologie, etc., le tout étant inséparable ${ }^{33}$ ». Cette formule implique que toutes les entités langagières, quel que soit leur niveau de structuration (des morphèmes aux textes) ont un caractère bi-face et relèvent des processus de sémiose ci-dessus résumés ; elle implique donc, contrairement à ce qu'impliquent les positions de Rastier ou Maingueneau, que les

31 Jean-Paul Bronckart, Ecaterina Bulea et Cristian Bota, Le projet de Ferdinand de Saussure, Genève, Droz, 2010.

32 Ferdinand de Saussure, Science du langage. De la double essence du langage, Genève, Droz, 2011 [édition établie par René Amacker].

33 Ferdinand de Saussure, De la double essence du langage, op. cit., p. 102. 
textes ont une essence et un mode de structuration qui sont radicalement « autres » que l'essence et les modes de structuration de la plupart des entités constitutives de leur entour.

Le second thème est celui du statut qu'il convient de donner à l'objet " langue ", dans ses rapports à la parole, au discours et/ou au texte, et sur ce thème les approches de Coseriu et de Saussure témoignent d'une convergence peu relevée mais néanmoins éclatante. Dans les conférences qu’il a données lors de son retour à Genève en 1891, Saussure, sur la base de ses multiples études antérieures portant sur l'évolution historique et le fonctionnement synchronique d'une trentaine d'idiomes, a proposé une première conception de l'objet de la linguistique qui est apparemment paradoxale en ce qu' elle pose l'existence d'une seule langue, qui a émergé avec l'espèce humaine et qui se continue en se transformant perpétuellement :

Il suffit d'y réfléchir un instant, puisque tout est contenu dans cette simple observation : chaque individu emploie le lendemain le même idiome qu'il parlait la veille et cela s'est toujours vu. Il n'y a donc eu aucun jour où on ait pu dresser l'acte de décès de la langue latine, et il n'y a eu également aucun jour où on ait pu enregistrer l'acte de naissance de la langue française. Il n'est jamais arrivé que les gens de France se soient réveillés, en se disant bonjour en français, après s'être endormis la veille en se disant bonne nuit en latin. ${ }^{34}$

$[\ldots]$ j'insisterais [...] encore une fois sur l'impossibilité radicale, non seulement de toute rupture, mais de tout soubresaut, dans la tradition continue de la langue depuis le premier jour même où une société humaine a parlé $[\ldots]{ }^{35}$

Cette première acception saussurienne de la langue correspond strictement à l'" activité de parler » dont Coseriu considérait qu'elle constituait l'objet général de la linguistique ; activité permanente eu égard à laquelle les langues naturelles constituent des " modalités historiques et géographiques ", c'est-à-dire des manières de parler qui, sous l'effet de la diffusion de ladite activité dans le temps et dans l'espace, n'exploitent que des sous-ensembles de ressources de la sémiose langagière. Pour Coseriu, cette langue/activité générale, ou langue saisie sous l'angle ontologique, est de l'ordre de l'energeia du triptyque de l'analyse aristotélicienne de l'activité.

Mais Saussure, propose une deuxième conception de la langue, qui est celle d'une puissance agentive associée à la conscience des sujets parlants, qui exerce des " responsabilités ", dont celles des processus de changement analogiques :

Une second fait, c'est que la langue a la conscience non seulement des éléments, mais aussi de <l'influence> qu'ils exercent les uns sur les autres quand on les place dans un certain ordre; la langue a le sentiment de leur sens logique, de leur ordre. ${ }^{36}$

34 Ferdinand de Saussure, Écrits de linguistique générale, Paris, Gallimard, 2002, p. 152.

35 Ferdinand de Saussure, Écrits de linguistique générale, op. cit., p. 163.

36 Eisuke Komatsu et George Wolf, Premier cours de linguistique générale (1907) d'après les cahiers d'Albert Riedlinger, Oxford/Tokyo, Pergamon, 1996, p. 96. 
$[\ldots]<$ la langue> passe son temps à interpréter et à décomposer ce qui est en elle de l'apport des générations précédentes - c'est là sa carrière ! - pour ensuite avec les sous-unités qu'elle a obtenues combiner de nouvelles constructions. ${ }^{37}$

Cette langue-puissance n'est cependant en réalité rien d'autre que le discours concrétisé dans les textes d'une langue naturelle, comme le confirme une note manuscrite désormais célèbre :

Toutes les modifications, soit phonétiques, soit grammaticales (analogiques) se font exclusivement dans le discursif. Il n'y a aucun moment où le sujet soumette à une révision le trésor mental de la langue qu'il a en lui, et crée à tête reposée des formes nouvelles (par ex. calmement [...]) qu'il se propose, \{promet\} de «placer» dans son prochain discours ${ }^{38}$.

Il apparaît donc que, contrairement à ce qui ressort du Cours de linguistique générale, la position effectivement défendue par Saussure est que si la parole comme acte factuel de verbalisation doit bien être distinguée de la langue, ce n'est nullement le cas de l'activité discursive concrétisée en textes ; activité qui relève, selon l'analyse de Coseriu, de la dynamis artistotélicienne.

Saussure propose encore une troisième conception de la langue, qui est celle des " états de langue ", en tant qu'arrangements et classements d'entités issues de la pratique discursive, mais ayant perdu au cours de ce transfert quelques propriétés spécifiques de cette dimension pratique :

[...] la langue entre d'abord dans notre esprit par le discursif, comme nous l'avons dit, et comme c'est forcé. Mais de même que le son d'un mot, qui est une chose entrée également dans notre for intérieur de cette façon, devient une impression complètement indépendante du discursif, de même notre esprit dégage tout le temps du discursif ce qu'il faut pour ne laisser que le mot. ${ }^{39}$

Alors que la langue-puissance est processus, cette langue-état est clairement de l'ordre du produit et correspond selon Coseriu au niveau aristotélicien de l'ergon. Mais cette langue-réceptacle a deux lieux distincts d'ancrage : d'un côté, les valeurs signifiantes issues de l'activité langagière se " déposent " dans le " cerveau " du sujet parlant (et constituent ce que nous qualifions pour notre part de " langue interne »), et d'un autre côté, elles sont recueillies, traitées et conceptualisée par certaines des instances collectives (et constituent ce que nous qualifions de "langue externe").

Nous présenterons, sous 4, les implications qu'ont ces deux thématiques pour l'élaboration d'un appareil conceptuel cohérent relatif aux textes et à leur entour.

37 Premier cours de linguistique générale, op. cit., p. 90.

38 Ferdinand de Saussure, Écrits de linguistique générale, Paris, Gallimard, 2002, p. 95.

39 Ferdinand de Saussure, "Ecrits", op. cit., p. 118. 


\section{Les apports de Volochinov}

Valentin Volochinov demeure un linguiste relativement peu connu parce qu'il a été victime de l'une des plus spectaculaires escroqueries intellectuelles $\mathrm{du} \mathrm{XX}^{\mathrm{e}}$ siècle, ayant visé à le dépouiller totalement de son œuvre au profit de Mikhaïl Bakhtine ${ }^{40}$. Dans ce qui suit, nous nous bornerons à évoquer quelques aspects de son positionnement épistémologique et de son approche des dimensions textuelles du langage.

L'objectif des travaux qu'il a entrepris de 1925 à 1930 était de jeter les bases d'une "poétique sociologique " qui se distinguerait de l'approche que développaient à la même époque les formalistes russes. Cette poétique devait à ses yeux prendre appui sur l'analyse des discours quotidiens, et ce pour deux raisons principales. Tout d'abord, dans la mesure où une même langue est utilisée, les discours quotidiens et les discours littéraires exploitent nécessairement les mêmes ressources (lexicales et morphosyntaxiques), et les processus mis en œuvre par les locuteurs ou les scripteurs, s'ils ne sont pas forcément identiques, présentent nécessairement des parentés qu'il conviendrait de mettre en évidence. Ensuite et surtout, les discours quotidiens sont plus étroitement articulés à leur contexte matériel et social, et constituent en conséquence un matériau privilégié pour l'examen des modalités d'interaction entre les propriétés (linguistiques) des discours et les éléments de leur entour :

Notre tâche est d'essayer de comprendre la forme de l'énoncé poétique comme forme d'une communication esthétique particulière qui se réalise dans le matériau verbal. Mais pour ce faire, il nous faudra examiner plus précisément certains aspects de l'énoncé verbal qui ne relèvent pas de l'art - dans le discours de la vie quotidienne -, car les fondements et les potentialités de la forme artistique ultérieure sont déjà posés dans ce type d'énoncé. L'essence sociale du mot apparaît ici plus clairement et plus nettement, et le lien qui unit l'énoncé au milieu social ambiant se prête plus facilement à l'analyse. ${ }^{41}$

Dans son examen des conditions de fonctionnement des discours quotidiens, Volochinov a souligné d'abord que la plupart d'entre eux ne peuvent être réellement (ou complètement) compris que lorsque sont connus les divers paramètres impliqués dans la situation de communication, et qu'en conséquence le contexte extra-verbal y aurait un statut analogue à celui du «sousentendu ", ou encore y jouerait un rôle équivalent à celui de la prémisse manquante dans un " enthymème" :

40 Voir Jean-Paul Bronckart et Cristian Bota, Bakhtine démasqué. Histoire d'un menteur, d'une escroquerie et d'un délire collectif, Genève, Droz, 2011.

41 Valentin Nicolaevitch Volochinov, "Le discours dans la vie et le discours en poésie ", in Mikhail Bakhtine le principe dialogique, Tzvetan Todorov (éd.), Paris, Éditions du Seuil, 1981, p. 188 ; édition originale en langue russe, 1926. 
Ainsi tout énoncé quotidien est un enthymème objectif et social. Il est comme un " mot de passe ", connu seulement de ceux qui appartiennent au même horizon social. C'est là la particularité des énoncés quotidiens : ils sont reliés par des milliers de fils au contexte vécu extra-verbal et, lorsqu'on les détache de ce contexte, ils perdent la quasi-totalité de leur sens; si l'on ignore le contexte vécu immédiat, on ne peut les comprendre. ${ }^{42}$

Ensuite, comme l'avait fait quelques années plus tôt son maitre Jakubinski, Volochinov a distingué trois dimensions constitutives de ce contexte :

- l'horizon spatio-temporel commun aux interlocuteurs ;

- la connaissance de la situation, également commune à ces interlocuteurs ;

- l'évaluation commune de la situation par ces mêmes interlocuteurs.

Il souligne enfin et surtout que le contexte ainsi défini ne peut nullement être considéré comme une force qui exercerait un effet direct et mécanique sur la teneur des énoncés ; pour lui, contexte et énoncé s'interpénètrent, ou sont dans un rapport de co-construction:

[...] il est parfaitement clair que le discours ne reflète pas ici la situation extraverbale comme le miroir reflète un objet. En l'occurrence il faut dire plutôt que le discours accomplit la situation, qu'il en dresse en quelque sorte le bilan évaluatif $[. .$.$] En sorte que la situation extra-verbale n'est en aucune façon la$ cause extérieure de l'énoncé, elle n'agit pas sur lui de l'extérieur comme une force mécanique. Non, la situation sintègre à l'énoncé comme un élément indispensable à sa constitution sémantique. ${ }^{43}$

Enfin, dans ses travaux d'analyse des textes profanes et littéraires, Volochinov a développé le thème du dialogisme, de l'attitude responsive active et de la polyphonie, et a proposé ce qui semble bien constituer la première formulation d'une méthodologie descendante d'analyse des « échanges verbaux concrets ", qui situe clairement la généricité au niveau des énoncés ou textes :

[...] l'ordre méthodologique à suivre pour étudier le langage doit être le suivant : (1) les formes et les types de l'interaction verbale en liaison avec les conditions concrètes dans lesquelles ces interactions se réalisent ; (2) les formes des énoncés singuliers, des interventions verbales singulières en liaison étroite avec l'interaction dont ils font partie, autrement dit les genres d'interventions verbales dans la vie et dans la création idéologique déterminés par l'interaction verbale ; (3) à partir de là, un réexamen des formes linguistiques telles qu'elles sont habituellement interprétées. ${ }^{44}$

42 Valentin Nicolaevitch Volochinov, "Le discours dans la vie et le discours en poésie ", op. cit., p. 192.

43 Valentin Nicolaevitch Volochinov, "Le discours dans la vie et le discours en poésie ", op. cit., p. 190-191.

44 Valentin Nicolaevitch Volochinov, Marxisme et philosophie du langage, Limoges, LambertLucas, 2010, p. 321. 


\section{Le texte, ses entours, et leurs modes d'interaction}

Nous proposerons dans ce qui suit l'appareil conceptuel que nous avons progressivement élaborée ${ }^{45}$, qui comporte un réseau de notions ayant trait aux niveaux de saisie des faits langagiers, un autre réseau ayant trait aux éléments de l'entour textuel, enfin une conception de la mise en interaction des faits langagiers et de leur entour.

\section{Les niveaux d'organisation du langagier}

- Conformément à la logique d'analyse " descendante " prônée par Volochinov, nous poserons que l'activité langagière universelle, ou " activité de parler ", constitue le premier niveau, ontologique ou de principe (energeia), de saisie des faits langagiers.

- Cette activité se déploie dans l'espace et dans le temps en mobilisant des sous-ensembles de ressources langagières universelles, en l'occurrence les procédés d'organisation discursive et les modes de stockage/classement des entités langagières en usage dans un idiome donné. Ce deuxième niveau de saisie est celui de la langue, dans sa dimension praxéologique (de puissance ou dynamis) comme dans sa dimension gnoséologique (de stockage/classement ou ergon).

- Le déploiement de l'activité langagière se concrétise en textes, ensembles bornés de productions mobilisant des unités et structures de la sémiose propres à une langue naturelle. Constituant le troisième niveau de saisie du langagier, les textes sont des unités dépendantes, en ce que leurs conditions d'ouverture et de fermeture sont déterminées par l'activité langagière elle-même (un texte n'a pas, en lui-même, de règles fixant ses conditions de démarrage et de clôture).

- Les textes ont pour fonction globale de commenter les activités ordinaires (ou non langagières), de contribuer à leur planification, à leur régulation, à l'évaluation de leurs effets, etc. Certaines de leurs propriétés internes sont dès lors nécessairement en rapport avec les composantes de l'entour textuel cf. 4.2. infra, pour une examen de ces composantes et se différencient en écho à leurs variations ; les textes se distribuent en conséquence en genres, c'est-à-dire en configurations textuelles plus ou moins adaptées au commentaire de telle ou telle activité pratique.

45 Voir Jean-Paul Bronckart, Activité langagière, textes et discours. Pour un interactionnisme sociodiscursif, Paris, Delachaux et Niestlé, 1997 ; Jean-Paul Bronckart, "Le langage au cœur du fonctionnement humain. Un essai d'intégration des apports de Volochinov, Vygotski et Saussure ", Estudos Linguisticos/Linguistic Studies, n 3, 2009, p. 31-62 ; Jean-Paul Bronckart, Daniel Bain, Bernard Schneuwly, Clairette Davaud et Auguste Pasquier, Le fonctionnement des discours. Un modèle psychologique et une méthode d'analyse, Paris, Delachaux et Niestlé, 1985. 
- Les exemplaires de genres textuels donnent naissance à des modèles génériques, qui sont décrits et étiquetés avec plus ou moins de précision, et qui s'organisent dans un espace théorique que nous qualifions d'architexte des différentes communautés verbales.

- Au sein des textes, on peut identifier des niveaux d'organisation infraordonnés que certains auteurs ont décrits en termes de " composantes sémantiques " (par exemple, la thématique, la dialectique, la dialogique et la tactique, pour Rastier ${ }^{46}$ ) ou en termes de types d'organisation séquentielle (chez Adam, la narration, la description, l'argumentation, l'explication et le dialogue). Nous considérons pour notre part que ce sont les « attitudes de locution » qu'évoquait Genette qui constituent les modes majeurs (parce que proprement sémiotiques) d'organisation infra-ordonnée aux textes ; modes que nous avons re-conceptualisés en termes de types de discours (discours interactif, discours théorique, récit interactif et narration). Ces types universels se traduisent, dans chaque langue naturelle, par des configurations d'unités et processus linguistiques relativement stables que nous avons décrites en détail pour le français ${ }^{47}$. Nous ne pourrons présenter ici notre approche de ces types discursifs, mais relèverons que leur fonction majeure est d'organiser la mise en interface du plan de la langue interne et des représentations individuelles avec celui de la langue externe et des représentations collectives.

- À ce qui précède s'ajoutent les niveaux de la syntaxique phrastique et de la morphologie.

\section{Les composantes de l'entour langagier}

L'entour langagier est constitué de deux ensembles d'entités dont le statut est fondamentalement différent : les préconstruits de l'histoire sociale humaine et les composantes des situations synchroniques de production langagière.

Les préconstruits de l'histoire sociale

Conformément à la perspective vygotskienne brièvement évoquée, il convient de prendre en considération d'abord des ensembles de produits de l'histoire culturelle des communautés humaines, au sein desquels on peut distinguer, d'un côté les préconstruits d'ordre praxéologique avec leurs soubassements sociaux ou sociopolitiques, et d'un autre côté les préconstruits gnoséologiques, les divers corpus de savoirs produits par les générations antérieures.

Les activités collectives non-verbales (ou activités pratiques) sont les plus évidents des préconstruits praxéologiques; orientées par des finalités diverses,

46 François Rastier, Arts et sciences du texte, Paris, PUF, 2001, p. 38-41.

47 Voir Jean-Paul Bronckart, Activité langagière, textes et discours. Pour un interactionnisme sociodiscursif, Paris, Delachaux et Niestlé, 1997. 
elles constituent les cadres organisant et médiatisant l'essentiel des rapports entre les individus et leur milieu. Les activités langagières sont articulées à ces activités pratiques dont elles assurent la planification, la régulation et souvent l'évaluation. La gestion de ces activités et de leurs produits s'effectue dans le cadre des diverses formations sociales, au sens donné à ce terme dans les textes marxiens ; formations génératrices de règles, de normes et de valeurs qui visent à expliciter les modalités acceptables de régulation des interactions entre membres des groupes et qui, en raison de la division du travail et des enjeux de pouvoir qu' elle entraîne, entretiennent des rapports toujours potentiellement conflictuels. Les formations sociales interviennent dans la gestion de l'ensemble des productions praxéologiques, y inclus les productions langagières, et il n'y a donc pas lieu de postuler l'existence de quelconques « formations discursives » qui en seraient distinctes.

Les préconstruits d'ordre gnoséologiques sont constitués des savoirs accumulés dans les traditions orales et écrites, qui ont été abstraits des contextes socioculturels et sémiotiques de leur élaboration pour s'organiser en systèmes de représentations collectives tendant à l'universalité. Adoptant les propositions d'Habermas ${ }^{48}$, nous distinguons trois ordres de savoir, ou mondes formels, se différenciant par la nature des objets qu'ils visent et par les critères de validité qui les régulent : le monde objectif en tant que système de savoirs s'adressant aux composantes de l'univers inscrites dans l'espace-temps (ou "physiques", au sens strict de ce terne), qui est évaluable en termes de véritél efficacité; le monde social en tant que système de savoirs s'adressant aux valeurs et aux modes acceptables d'interaction entre les humains, qui est évaluable en termes de rapport aux normes; le monde subjectif en tant que système de savoirs s'adressant à l'intériorité psychique des personnes, qui est évaluable en termes de sincérité/authenticité. Dans ce cadre, les savoirs élaborés à propos du langagier, tels qu'ils sont codifiés dans la "langue externe », constituent une des rubriques du monde objectif.

\section{Les situations de production langagière}

La production langagière est une action ${ }^{49}$ certes de la responsabilité d'un actant doté de représentations, mais qui se déploie dans un environnement constitué d'entités définissables indépendamment desdites représentations. Cet environnement, que nous qualifions de "situation de production ", comporte deux ensembles d'entités susceptibles d'influer sur les propriétés de la production verbale.

48 Jürgen Habermas, Théorie de l'agir communicationnel, t. 1, Paris, Fayard, 1987.

49 Nous qualifions d'" activité langagière " les pratiques verbales se déployant, en principe ou concrètement, au plan collectif, et nous qualifions d'" action langagière " une intervention verbale imputable à une personne singulière. 
Le premier ensemble est constitué d'entités " physiques " découlant du fait que toute production constitue un comportement concret, accompli par un actant situé dans les coordonnées de l'espace et du temps. Ce registre de la situation de production comporte les cinq entités de fait impliquées dans l'appareil formel de l'énonciation conceptualisé par Benveniste, à savoir :

- l'émetteur, comme personne (ou machine) qui produit physiquement un texte ;

- le(s) récepteur(s), comme entité quelconque susceptible(s) de recevoir concrètement ce texte ;

- le lieu de production du texte ;

- le moment de production du texte ;

- toute entité externe accessible dans l'espace-temps de production du texte.

Le second ensemble a trait au fait que toute production langagière s'inscrit aussi dans le cadre d'une formation sociale, et plus précisément dans une forme d'interaction communicative impliquant le monde social (normes, valeurs, règles, etc.) et le monde subjectif (image que l'on donne de soi en agissant). Ce registre socio-subjectif de la situation de production peut être décomposé en quatre paramètres majeurs :

- le lieu social, notion englobant la formation sociale, l'institution, ou plus simplement le mode d'interaction dans le cadre duquel est produit un texte ;

- le rôle social joué par l'émetteur dans l'interaction en cours (qui lui donne son statut d'énonciateur);

- le rôle social attribué au récepteur (qui lui donne son statut de destinataire);

- le (ou les) but(s) du texte, au sens d'effet que celui-ci est susceptible de produire sur le destinataire.

\section{Les interactions génératrices des textes}

Comme Kleiber ${ }^{50}$ l'avait analysé avec précision, il convient de ne pas confondre les registres situationnels évoqués ci-dessus (ou "sites " dans les termes de l'auteur) qui pré-existent en tant que tels, et les représentations que s'en construit un actant verbal.

Lors de la production d'un texte, cet actant va nécessairement mobiliser ses représentations propres ayant trait la dimension physique de la situation de production, et il va mobiliser également ses représentations des dimensions sociales de la situation qui, en partie au moins, sont dépendantes de ses connaissances ayant trait aux dimensions praxéologiques de l'entour langagier (quelle est l'activité pratique concernée ?) et à ses dimensions sociologiques (quelle est la formations sociale impliquée et quels sont les enjeux sociaux de l'intervention ?). Et c'est cet ensemble représentatif complexe qui constitue le

50 George Kleiber, «Contexte, interprétation et mémoire : approche standard vs approche cognitive ", Langages, n 103, 1994, p. 9-22. 
contexte véritable de la production d'un texte, contexte exerçant un contrôle sur certains aspects illocutoires de l'organisation dudit texte.

L'actant va mobiliser également ses représentations relatives aux mondes formels pour élaborer le contenu thématique de son intervention verbale, et cet autre réseau de connaissances interviendra donc dans la construction des aspects locutoires de l'organisation du texte.

Ces deux ensembles de représentations ne constituent pas pour autant des facteurs causaux, susceptibles d'exercer des effets directs ou mécaniques sur les propriétés des textes, mais constituent plutôt une base d'orientation à partir de laquelle un ensemble de "décisions " doivent être prises. Celles-ci consistent à choisir, parmi les modèles génériques disponibles dans l'intertexte, le genre de texte qui paraîtra le plus adapté aux caractéristiques de la situation de production, telles que l'actant se les représente, et à effectuer également les choix de types de discours et des diverses structures constitutives de la textualité.

Depuis la base d'orientation, se met ainsi en ouvre un processus d'emprunt qui comporte deux mouvements : d'une part il s'agit donc d'adopter un des modèles de genre disponibles dans l'intertexte ; d'autre part, dans la mesure où les valeurs du contexte et du contenu thématique d'une action langagière sont toujours en partie nouvelles, il s'agit aussi d'adapter le modèle générique choisi à ces valeurs particulières.

Si le mouvement d'adoption donne ainsi au texte sa dimension générique, le mouvement d'adaptation lui donne une part de sa dimension stylistique ; et plus généralement, le processus d'adoption-adaptation génère lui-même de nouveaux exemplaires de genres, plus ou moins différents des exemplaires préexistants. Et c'est par l'accumulation de ces processus singuliers d'emprunt que les genres se modifient en permanence et contribuent ainsi à la dynamique ininterrompue du déploiement de l'activité langagière.

Jean-Paul Bronckart

Groupe de Recherche pour l'Analyse du Français Enseigné (GRAFE)

Université de Genève Jean-Paul.Bronckart@unige.ch 